\section{Assistência pública de saúde no contexto da transição demográfica brasileira: exigências atuais e futuras}

\author{
The public healthcare system in the context \\ of Brazil's demographic transition: current \\ and future demands
}

Antonio da Cruz Gouveia Mendes 1
Domicio Aurélio de Sá 1
Gabriella Morais Duarte Miranda 1
Tereza Maciel Lyra 1
Ricardo Antonio Wanderley Tavares ${ }^{2}$

\section{Introdução}

This paper assesses inpatient and outpatient care and their capacity to respond to changing demands in the context of the demographic transition in Brazil. The data were obtained from studies by the Brazilian Institute of Geography and Statistics (IBGE) and databases in the National Health System (CNES, SIH, and SIA). The reduction in birth, fertility, and infant mortality rates and the increase in life expectancy at birth are still driving population growth, while decreas ing the dependency rate, thereby providing the opportunity to make necessary adjustments. The population increased by more than 27.5 million from 1999 to 2009, with a 26.7\% reduction in hospital beds and 947,000 hospitalizations, with distortions in the distribution by specialty, but with increases in high-complexity outpatient and inpatient care. The results show that Brazil is undergoing a transition in the healthcare model, requiring greater capacity for future planning of a more complex system and revising the model to prepare for a larger elderly population in the coming decades.

Delivery of Health Care; Demographic Transition; Health Services Needs and Demand
No início da década de 70 do século passado, no calor da discussão sobre as políticas de planejamento familiar, Yunes 1, em seu estudo sobre a dinâmica populacional, conclui que o crescimento populacional acelerado é consequência e não causa do subdesenvolvimento e como tal não é problema médico. A discussão das características demográficas da época e a adoção de políticas públicas de saúde tinham como centro do debate a questão do planejamento familiar, o acesso a métodos contraceptivos e a defesa da soberania nacional na adoção de políticas de desenvolvimento social.

Desde então, a sociedade brasileira mudou, os avanços conquistados na política de saúde como a garantia constitucional do direito à saúde, a constituição do Sistema Único de Saúde (SUS) e uma maior prioridade para a atenção primária, com ênfase na assistência à saúde materna e infantil contribuíram para o aumento da expectativa de vida e para a redução das taxas de mortalidade, em particular da mortalidade infantil 2.

Quatro décadas depois, passa a fazer parte da agenda o crescimento da população produtiva e idosa, a mudança do perfil epidemiológico e as novas necessidades de saúde da população que exigem a adoção de novas políticas públicas de saúde. Enquanto em meados do século XX se discutiam as causas e consequências do crescimento populacional, no início do século XXI a 
pauta são as causas e consequências da transição demográfica. A chamada "bomba demográfica" já foi desativada, do ponto de vista da estatística populacional, a maior mudança foi a universalização do processo de transição demográfica ${ }^{3}$.

Este estudo pretende avaliar a oferta e produção assistencial hospitalar e ambulatorial, as suas transformações e sua capacidade de responder às novas exigências, atuais e futuras neste contexto da transição demográfica brasileira.

\section{Metodologia}

Trata-se de um de estudo descritivo de âmbito nacional, com base em dados secundários do Instituto Brasileiro de Geografia e Estatística (IBGE) e do Ministério da Saúde, tendo como período de referência a série histórica de 1999 a 2009.

As informações demográficas relativas à população brasileira, taxa de fecundidade total, taxa de natalidade, taxa de mortalidade infantil, taxa média geométrica de crescimento anual e esperança de vida ao nascer foram obtidas em estudo de projeção da população do país, realizada pelo IBGE 4 .

Para obtenção dos dados assistenciais, foram utilizados os sistemas de informações assistenciais do SUS. Esses sistemas de informações vêm sendo amplamente utilizados na avaliação de desempenho da rede assistencial quanto aos gastos, à oferta de serviços, à demanda hospitalar, à cobertura assistencial, entre outros, e como informação complementar na vigilância à saúde. Além de possibilitar, devido à multiplicidade de variáveis disponíveis, a construção de indicadores simples e compostos de acordo com as necessidades efetivas do que se quer avaliar 5,6,7.

O número de leitos hospitalares disponíveis para o SUS foi obtido dos cadastros mensais do Ministério da Saúde, sendo construída a média anual. Para o período de 1999 a 2004 foram utilizados os dados registrados nos cadastros extintos do SUS (Cadastro Hospitalar - $\mathrm{CH}$ ), disponibilizados pelo Departamento de Informática do SUS (DATASUS). E para os demais anos da série (2005 a 2009) foi utilizado o Cadastro Nacional de Estabelecimentos de Saúde (CNES).

As internações realizadas foram obtidas do total de Autorizações de Internação Hospitalar (AIH) pagas, registradas no Sistema de Informações Hospitalares do SUS (SIH/SUS), e os procedimentos ambulatoriais foram analisados com uso do Sistema de Informações Ambulatoriais do SUS (SIA/SUS). Todos os arquivos foram processados pelo software TabWin (DATASUS. http:// portal.saude.gov.br/portal/se/datasus/area. cfm?id_area=732), sendo necessária a criação de arquivos de definição e conversão para facilitar a elaboração das tabelas.

$\mathrm{Na}$ análise dos procedimentos ambulatoriais, foi excluído o grupo de medicamentos, tanto no geral como na alta complexidade, por representar a quantidade de medicamentos excepcionais dispensados e não procedimentos realizados. Para compor a alta complexidade ambulatorial, foram utilizados os grupos de procedimentos que representaram esse nível de complexidade em todo o período analisado: tratamento em nefrologia (terapia renal substitutiva), tratamento em oncologia (quimioterapia e radioterapia), ressonância magnética, tomografia computadorizada, medicina nuclear - in vivo, radiologia intervencionista e hemodinâmica.

\section{Resultados e discussão}

\section{O contexto da transição demográfica no país}

Em 1988, Veras 8 já identificava para as políticas públicas os desafios que seriam enfrentar por um longo período uma grande demanda por políticas de educação e médico-sociais para a parcela jovem da população convivendo com o crescimento da população idosa e as suas necessidades médico-sociais.

Nas décadas de 80 e 90, o Brasil passou a ter uma redução mais acelerada da taxa de natalidade que as taxas de mortalidade infantil e de fecundidade. Porém, em 2004 a taxa de fecundidade atinge o nível de reposição da população, 2,1 filhos por mulher (Tabela 1). Essas reduções, especialmente das taxas de fecundidade, de natalidade e mortalidade, levam a um menor ingresso de jovens na população que passam a viver períodos mais longos, processo gradativo conhecido como "transição demográfica" 9.

O declínio da fecundidade de certa forma permite ao Estado atender de modo mais adequado às demandas sociais da população. No entanto, 0 contexto de desigualdade e velocidade com que ocorrem tais transformações no Brasil apontam para a complexidade crescente nas alternativas de atenção às necessidades dessa nova estrutura etária emergente 10.

Além disso, ocorre uma significativa redução da mortalidade infantil e um crescimento significativo da esperança de vida ao nascer do brasileiro. A mortalidade infantil reduziu de 69,10 por mil nascidos vivos em 1980 para 22,5 por mil em 2009, enquanto a esperança de vida ao nascer aumentou de 62,6 anos em 1980 para 73,09 anos em 2009. Mesmo com as reduções das taxas de natalidade, de fecundidade e de mortalidade 
Taxas de fecundidade total, natalidade, mortalidade infantil, de crescimento, esperança de vida, população, leitos e internações no SUS no Brasil entre 1999 e 2009 e estimativas para 2040.

\begin{tabular}{|c|c|c|c|c|c|c|c|c|}
\hline Ano & $\begin{array}{c}\text { Taxa de } \\
\text { fecundidade } \\
\text { total }\end{array}$ & $\begin{array}{c}\text { Taxa de } \\
\text { natalidade }\end{array}$ & $\begin{array}{c}\text { Taxa de } \\
\text { mortalidade } \\
\text { infantil }\end{array}$ & $\begin{array}{c}\text { Taxa } \\
\text { crescimento } \\
\text { anual (\%) }\end{array}$ & $\begin{array}{c}\text { Esperança } \\
\text { de vida ao } \\
\text { nascer }\end{array}$ & População & Leitos & Internações \\
\hline 1980 & 4,06 & 32,13 & 69,10 & 1,930 & 62,60 & 118.562 .549 & & \\
\hline 1990 & 2,79 & 24,21 & 47,00 & 1,786 & 66,57 & 144.090 .756 & & \\
\hline 1999 & 2,41 & 21,30 & 31,70 & 1,493 & 70,02 & 163.947 .436 & 501.731 & 12.438 .376 \\
\hline 2000 & 2,39 & 21,13 & 30,10 & 1,486 & 70,43 & 169.799 .170 & 498.001 & 12.426 .137 \\
\hline 2001 & 2,34 & 20,84 & 29,20 & 1,465 & 70,71 & 172.385 .776 & 497.536 & 12.227 .236 \\
\hline 2002 & 2,27 & 20,33 & 28,40 & 1,426 & 71,00 & 174.632 .932 & 464.059 & 12.233 .702 \\
\hline 2003 & 2,20 & 19,76 & 27,50 & 1,373 & 71,29 & 176.876 .251 & 454.034 & 12.094 .875 \\
\hline 2004 & 2,13 & 19,12 & 26,60 & 1,314 & 71,59 & 179.108 .134 & 361.612 & 11.953 .856 \\
\hline 2005 & 2,06 & 18,45 & 25,80 & 1,250 & 71,88 & 184.184 .074 & 375.664 & 11.861 .494 \\
\hline 2006 & 1,99 & 17,75 & 25,00 & 1,182 & 72,18 & 186.770 .613 & 373.421 & 11.721 .412 \\
\hline 2007 & 1,93 & 17,06 & 24,10 & 1,113 & 72,48 & 189.335 .191 & 374.467 & 11.739 .258 \\
\hline 2008 & 1,86 & 16,38 & 23,30 & 1,045 & 72,78 & 189.612 .814 & 387.038 & 11.107.155 \\
\hline 2009 & 1,81 & 15,77 & 22,50 & 0,980 & 73,09 & 191.481 .045 & 367.974 & 11.491 .013 \\
\hline 2040 & 1,50 & 8,91 & 8,20 & $-0,023$ & 79,95 & 219.075 .130 & & \\
\hline
\end{tabular}

Fonte: Instituto Brasileiro de Geografia e Estatística; Sistema de Informações Hospitalares, Ministério da Saúde.

infantil e o aumento da expectativa de vida ao nascer, o Brasil ainda apresenta um crescimento demográfico, embora com uma tendência de redução das taxas de crescimentos anuais. Em 2009, pela primeira vez, a taxa de crescimento nacional foi inferior a $1 \%(0,98 \%)$. As projeções demográficas indicam que, caso seja mantida tal tendência, a partir de 2040 as taxas de crescimento serão negativas, iniciando assim um processo de redução da população brasileira, aspecto que lança significativos desafios aos gestores públicos (Tabela 1).

A Figura 1 mostra a evolução das faixas etárias populacionais nas décadas entre 1990 e 2040 . No geral, percebe-se uma efetiva redução da população com menos 15 anos, o aumento da população idosa (mais de 60 anos) e uma estabilização da faixa etária entre 15 a 60 anos.

Em 1990 eram mais de 50,4 milhões de crianças com menos de 15 anos (35\% da população), passando para 49,4 milhões (25,6\%), em 2010 e, pelas projeções, em 2040 serão 32,6 milhões, representando apenas $14,9 \%$ da população brasileira.

A população entre 15 e 60 anos, continuará crescendo em termos absolutos e relativos até a década entre 2020, quando alcança $66 \%$ do total da população. A partir de 2030 o peso relativo deste grupo etário cai, chegando a $61 \%$ em 2040 .
A população idosa que já apresentava um crescimento entre 1990 (7,2\%) e 2010 (10\%), terá uma maior intensidade de crescimento a partir de 2020 , passando de 28,3 milhões $(13,7 \%)$ para 52 milhões $(23,8 \%)$ de idosos em 2040, quase um quarto do total de habitantes do país.

Portanto, esses resultados demonstram que até a década de 20 do século XXI o Brasil terá uma estruturação etária da população com o crescimento e predomínio da população adulta, a redução da população infantil e um crescimento da população idosa, mas sem grande significância no total da população. No entanto, de acordo com as estimativas, a partir da década de 30 haverá uma inversão, com uma diminuição paulatina da população em idade produtiva e o predomínio de idosos em relação ao número de crianças.

Esses dados e projeções apontam de um lado grandes desafios e de outro, grandes oportunidades para o planejamento e gestão de políticas públicas, em particular as políticas sociais. Frente às mudanças, há a necessidade de estruturação de serviços e de programas de saúde que possam responder às demandas emergentes do novo perfil epidemiológico do país. Os idosos utilizam os serviços hospitalares de maneira mais intensiva que os demais grupos etários, envolvendo maiores custos, implicando tratamento de duração mais prolongada e de recuperação mais lenta e complicada 11 . 


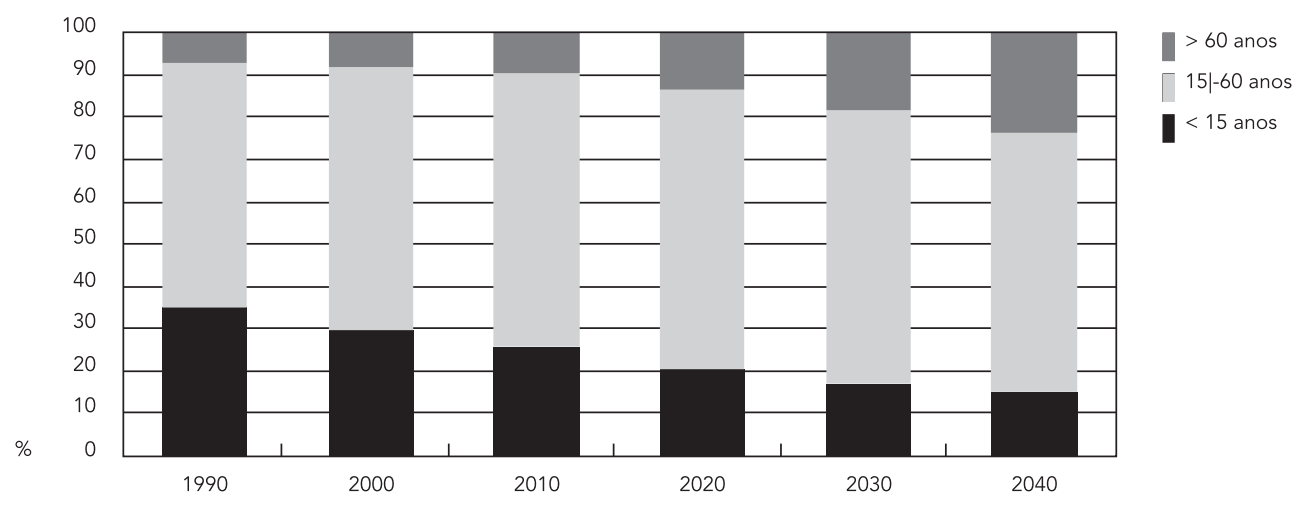

Fonte: Instituto Brasileiro de Geografia e Estatística.

O quadro demográfico atual de redução da população menor de 15 anos, o pequeno crescimento da população idosa e um grande crescimento da população produtiva traz como consequência um maior contingente de pessoas disponível para trabalhar e gerar renda para família e para o país. Essas características da fase atual de nossa transição demográfica brasileira possibilitam tirar proveitos da redução da taxa de dependência (aumento relativo da população em idade de trabalhar) para promover ajustes necessários e assim enfrentar a fase seguinte em décadas futuras. É o que se chama de "bônus demográfico" ou "dividendo demográfico" 3. Mas nada adiantará se o país não souber captar tal momento, gerando os empregos suficientes e não melhorar os atuais níveis de escolaridade desses jovens que ingressam na vida adulta. A combinação do desemprego com a baixa escolaridade resulta no aumento da marginalidade e da violência no país. Num outro extremo dos cenários possíveis, tal bônus demográfico somado a uma maior inserção das mulheres no mercado de trabalho (46,7\% em 2007) 12 poderá abrir nas próximas décadas oportunidades demográficas que podem ser usufruídas pela sociedade e economia. Para Brito 13, a transição demográfica está longe de ser considerada neutra: pode tanto criar possibilidades demográficas que potencializem o crescimento da economia e do bem-estar social da população, quanto ampliar as graves desigualdades sociais que marcam a sociedade brasileira.

A sociedade necessita investir na atual geração de crianças, particularmente nas áreas de saúde e educação. Não se trata de garantir, apenas, a melhoria da qualidade de vida dessas gerações, mas também o equilíbrio de toda a sociedade. Dependerá das atuais gerações de jovens a garantia, em médio e longo prazos, de ofertarem uma vida digna às gerações de idosos. Ademais, é nesta fase que a sociedade deve se preparar, mediante reformas institucionais na área da seguridade social, para conviver, no futuro próximo, com altas, porém sustentadas taxas de dependência de idosos 14 .

\section{Assistência ambulatorial e hospitalar neste contexto demográfico}

Na análise da assistência hospitalar nacional, por meio do número de leitos e de internações realizadas, de acordo com os dados registrados SIH/SUS e no CNES entre os anos de 1999 e 2009 (Tabela 1), observa-se uma redução de $26,6 \%$ no número total de leitos e 7,6\% das internações, representando uma diminuição de 947.363 internações hospitalares. Entretanto, no mesmo período, ocorreu crescimento populacional de $16,8 \%$, ou seja, mais de 27,5 milhões de habitantes.

Desse modo, se se levar em consideração que $25,9 \%$ da população são cobertos por planos de saúde 15 e se utilizar o parâmetro de necessidade média de 2,75leitos por mil habitantes, segundo a Portaria GM/MS no . 1.101/2002 16, constatar-se-á que, em 1999, havia um excedente de 167.647 leitos públicos no país. Com a implantação do CNES, em 2003, aconteceu uma reformulação dos instrumentos e dos processos de cadastramento da rede de saúde, ocorrendo uma drástica 
redução de 47.697 leitos disponíveis para o SUS. Assim, de acordo com este parâmetro, em 2009, o déficit era de 22.216 leitos, considerando os $74,1 \%$ da população que dependem exclusivamente do SUS.

Em relação às internações, a redução entre 2007 e 2008 foi de 632.103 internações, justificadas em parte pela mudança gerada na implantação da nova tabela de procedimentos do SUS. A tabela unificada redirecionou a lógica global da codificação dos procedimentos, estando mais adequada aos processos de programação, análise de informação e controle, avaliação e auditoria 17. De acordo com os parâmetros assistenciais 16 , para $74,1 \%$ da população brasileira, em 2009, seriam necessárias 11.350 milhões de internações/ano, portanto, houve um excedente de 140 mil. Contudo, seria deficitário em 3,8 milhões de internações, se considerássemos o universo da população, sobretudo em função do grande número de internações de alta complexidade realizadas no SUS pela população coberta por planos de saúde.

Para analisar este comportamento, temos de levar em conta movimentos de sentidos contrários. Primeiro, a evolução tecnológica da prática médica que leva a uma constante substituição de procedimentos hospitalares por ambulatoriais e a redução do número de dias de internamentos necessários, tendo como consequência a redução das internações e do tempo de permanência nos hospitais. Segundo, tal evolução tecnológica gera necessidades crescentes, pressão do mercado para a venda de equipamentos e o consu- mo de serviços. Terceiro, temos o crescimento e envelhecimento da população, além do aumento do numero de acidentes e violências no país. Logo, a resultante esperada desses movimentos é o crescimento e complexidade da assistência hospitalar.

Ao analisar a oferta de leitos, o número de internações realizadas por especialidades clínicas e comparando com as populações nos anos de 1999 e 2009 (Tabelas 2 e 3), verificamos um comportamento não uniforme em relação às necessidades.

Na clínica médica, percebe-se uma redução de 4,5\% no número de internações e uma redução de 27,6\% no número de leitos. Apesar dos avanços clínicos, que permitem hoje o tratamento ambulatorial ou domiciliar para um grande número de patologias que antes tinham resolução hospitalar, esses resultados não são compatíveis com o crescimento de 28,3 milhões da população adulta, o seu envelhecimento no período e a consequente necessidade de internações com um grau de complexidade maior.

Além da redução geral, as internações na clínica médica apresentaram números praticamente iguais para a população idosa, quando ocorreu, no período, um acréscimo de 6,58 milhões de idosos (51,3\%). Ou seja, em 1999 ocorreram 124,8 internações por habitantes com idade maior que 60 anos, havendo uma redução para 84,8 em 2009 (Tabela 3), quando, esperavase que essa população demandasse um maior número de cuidados tanto ambulatoriais quanto hospitalares.

Tabela 2

Leitos, internações e percentual por especialidades médicas no SUS em 1999 e 2009.

\begin{tabular}{|c|c|c|c|c|c|c|}
\hline \multirow[t]{2}{*}{ Especialidades } & \multicolumn{3}{|c|}{1999} & \multicolumn{3}{|c|}{2009} \\
\hline & Leitos & Internações & $\%$ & Leitos & Internações & $\%$ \\
\hline Clínica médica & 147.665 & 4.175 .624 & 33,6 & 106.899 & 3.987 .626 & 34,7 \\
\hline Clínica cirúrgica & 94.125 & 2.596 .390 & 20,9 & 76.822 & 3.198 .391 & 27,8 \\
\hline Obstetrícia & 72.145 & 2.998 .767 & 24,1 & 47.857 & 2.163 .655 & 18,8 \\
\hline Pediatria & 81.485 & 1.716 .296 & 13,8 & 53.860 & 1.429 .567 & 12,4 \\
\hline Psiquiatria & 72.807 & 827.064 & 6,6 & 42.799 & 537.460 & 4,7 \\
\hline Cuidados prolongados & 16.736 & 81.241 & 0,7 & 9.188 & 88.135 & 0,8 \\
\hline Tisiologia & 2.786 & 20.818 & 0,2 & 1.900 & 12.738 & 0,1 \\
\hline Reabilitação & 824 & 4.903 & 0,0 & 1.024 & 14.971 & 0,1 \\
\hline Leitos hospital-dia & 2.049 & 17.273 & 0,1 & 4.776 & 58.470 & 0,5 \\
\hline Leitos UTI & 11.110 & - & 0,0 & 14.738 & - & 0,0 \\
\hline Outros & - & - & 0,0 & 8.112 & - & 0,0 \\
\hline Total & 501.731 & 12.438 .376 & 100,0 & 367.974 & 11.491 .013 & 100,0 \\
\hline
\end{tabular}

Fonte: Sistema de Informações Hospitalares, Ministério da Saúde; Cadastro Nacional de Estabelecimentos de Saúde. 
Leitos e internações por grupos populacionais no SUS em 1999 e 2009

\begin{tabular}{lcc}
\hline Grupos populacionais/leitos/internação & 1999 & 2009 \\
\hline População até 14 anos & 51.746 .334 & 49.138 .121 \\
Leitos em pediatria & 81.485 & 53.860 \\
Internações em pediatria & 1.701 .037 & 1.398 .557 \\
População com 15 anos e mais & 111.770 .763 & 142.342 .924 \\
Leitos em clínica médica & 147.665 & 106.899 \\
Internações em clínica médica & 3.850 .468 & 3.796 .704 \\
População com 60 anos e mais & 12.844 .001 & 19.428 .086 \\
Leitos em clínica médica & 147.665 & 106.899 \\
Internações em clínica médica & 1.602 .774 & 1.647 .001 \\
População de mulheres de 10 a 49 anos & 53.663 .899 & 61.417 .666 \\
Leitos em obstetrícia & 72.145 & 47.857 \\
Internações em obstetrícia & 2.998 .767 & 2.163 .655 \\
\hline
\end{tabular}

Fonte: Instituto Brasileiro de Geografia e Estatística; Sistema de Informações Hospitalares, Ministério da Saúde;

Cadastro Nacional de Estabelecimentos de Saúde.

Em relação à clínica obstétrica, deu-se uma redução de 33,7\% no número de leitos e 27,8\% das internações (Tabela 2). Entre estas internações obstétricas, as que tiveram como procedimento a realização do parto, reduziram de 2.653 milhões em 1999 para 1.997 milhões em 2009, redução de 32,8\%. Embora havendo, entre 1999 e 2009, um crescimento $14,5 \%$ da população de mulheres em idade fértil (10 a 49 anos) (Tabela 3), o país se encontra num processo de transição demográfica, com redução das taxas de fecundidade e natalidade, o que leva a um decréscimo do número de partos. Os registros do Sistema de Informações sobre Nascidos Vivos (SINASC; http://www.tabnet.datasus.gov.br/cgi/tabcgi. exe?sinasc/cnv/nv, acessado em 11/Ago/2010) corroboram tal fato, pois em 1999 foram 3.256 milhões de nascidos vivos, enquanto em 2007 os nascidos vivos totalizaram 2.891 milhões, uma redução de $11,21 \%$. Vale ressaltar que a maior redução do número de partos aconteceu na rede pública, apontando maior participação da assistência privada ao parto, fato que, somado às mudanças demográficas, contribui para explicar a redução das internações obstétricas no SUS.

Entre 1999 e 2009, existiu uma redução de 286.729 internações pediátricas (16,7\%) e 33,9\% no número de leitos, ao passo que a redução da população menor de 15 anos foi de apenas $5,0 \%$. Nesse período aconteceu uma melhoria das condições epidemiológicas com redução da mortalidade infantil no país e uma ampliação da oferta de ações e serviços de atenção primária à saúde, que resolve muitos agravos no nível ambulatorial, ainda que também possa ampliar a porta de entrada no sistema de saúde, propiciando a identificação de novas necessidades de hospitalização. Portanto, essa grande redução de internações em relação à pequena redução da população pediátrica ou representa uma desassistência hospitalar ou uma melhoria das condições epidemiológicas, sociais e da assistência ambulatorial que de fato impactaram na situação de saúde da população, reduzindo assim o número de internações, o que parece ser a explicação mais coerente com tais transformações.

Para as demais especialidades, verifica-se maior coerência evolutiva (Tabela 2), sendo compatível com o contexto nacional de transição demográfica, epidemiológica e político-assistencial. Na clínica psiquiátrica, por exemplo, houve uma redução de $41,2 \%$ no número de leitos e 35,1\% nas internações, situação compatível com o novo enfoque da política de saúde mental, com base nos princípios da reforma psiquiátrica, que privilegia o tratamento ambulatorial numa perspectiva humanizadora e integradora dos indivíduos à família e à sociedade.

Os dados identificados para a clínica cirúrgica demonstram que aconteceu uma readequação ao novo momento que o país atravessa. O número de internações cirúrgicas cresceu $23,2 \%$, compatível com o crescimento de $16,8 \%$ da população, apesar de ter havido uma redução de $18,4 \%$ no número de leitos, provavelmente em virtude da redução do tempo médio de permanência no hospital para cada internação. 
O crescimento do número de leitos da reabilitação em $24,3 \%$ e das internações nessa especialidade em $238,5 \%$ e, sobretudo, o aumento do número de leitos de Unidade de Terapia Intensiva (UTI), passando de 11.110 para 14.738 leitos, aumento de $32,6 \%$, demonstra que sucedeu um aumento da complexidade hospitalar no intervalo de 1999 a 2009. Por mais que essa oferta de UTI ainda esteja aquém da necessidade, que seria de 29.488 leitos de UTI para $80 \%$ da população brasileira 16, atendeu. Há a necessidade de dobrar o número de leitos de UTI. Embora a oferta de serviços influencie o seu consumo, como ficou evidenciado em estudo que demonstrou que quanto maior o número de leitos hospitalares disponíveis, maior a chance de o indivíduo se internar, caracterizando-se como uma demanda induzida pela oferta 18 .

Resultado semelhante foi obtido em avaliação dos cuidados de saúde aos norte-americanos com idade igual ou superior a 65 anos por intermédio do programa Medicare. Na avaliação, foi constatado que as variações em relação aos atendimentos em hospitais de referência, regiões, gasto per $c a$ pita e alocação de recursos não eram explicadas pela doença ou preferência do paciente, portanto não estão associadas ao fornecimento de cuidados mais eficazes. A melhor utilização está associada ao grau de organização e coordenação dos cuidados à saúde em regiões onde, por exemplo, há mais médicos de cuidados primários à saúde 19 .

Esse crescimento da complexidade da assistência também é evidenciado quando analisamos os procedimentos de alta complexidade realizados pelo SUS. No período de 1999 a 2009, encontra-se (Figura 2) um significativo aumento da assistência de alta complexidade tanto ambulatorial quanto hospitalar. Na assistência ambulatorial de alta complexidade, os procedimentos comuns no período estudado apresentaram um crescimento de $71,3 \%$, passando de $13,41 \mathrm{mi}-$ lhões para 22,98 milhões de procedimentos de alta complexidade. $\mathrm{O}$ incremento foi, particularmente, decorrente das terapias renal substitutiva e oncológicas (quimio/radioterapia), e, em menor grau, dos exames complementares de alta complexidade (tomografia, ressonância magnética, medicina nuclear, radiologia intervencionista e hemodinâmica).

Na atenção hospitalar de alta complexidade, as internações passaram de 254.699 para 516.999 , um crescimento de $103 \%$ entre 1999 e 2009. Quando se relativiza com a população, percebese que havia 1,55 internação de alta complexidade por mil habitantes em 1999, passando para 2,70/1.000 em 2009. Esses resultados confirmam que há um processo de sofisticação da assistência hospitalar no SUS, corroborando estudo an- terior sobre a assistência de alta complexidade nas doenças crônicas não transmissíveis 20 .

Em projeções realizadas levando-se em consideração o envelhecimento da população e a variação do nível da renda do país até meados do século XXI (tendo em conta um crescimento médio do rendimento per capita de 2,5\% ao ano), a demanda por serviços de saúde para consultas médicas deverá crescer 59\%, para exames, 96\% para tratamentos, $122 \%$, e para internações, $39 \%$. Em relação ao gasto com saúde, o envelhecimento da população deverá acarretar um aumento do gasto com saúde em relação ao PIB de aproximadamente $30 \%$ até 2050 , dos atuais $8,2 \%$ para $10,7 \%$. Tais projeções devem ser entendidas como uma sinalização das mudanças, que estão em curso no Brasil, e servir de suporte para o planejamento e para a adaptação da oferta à demanda por serviços de saúde no país 21 .

\section{Considerações finais}

O processo de mudanças da assistência na rede pública de saúde do país ocorrida nos últimos dez anos é caracterizado por quatro aspectos principais: grande redução no número de internações, ampliação da assistência ambulatorial, crescimento da assistência de alta complexidade ambulatorial e hospitalar e uma distribuição dos leitos e das internações nas especialidades não racional em relação às necessidades da população. Tal processo apresenta algumas características que demonstram ser um movimento não planejado de acordo com as transformações exigidas pelo momento de transição demográfica, social e epidemiológica e uma distribuição não racional das internações nas especialidades.

A relação entre consumo de serviços de saúde e as condições sociais e geográficas já vem sendo estudada. Travassos et al. 22, tendo como fonte de dados o SIH/SUS, estudaram a desigualdade no uso de serviços de saúde pelas dimensões geográficas e sociais. Os resultados demonstraram uma multiplicidade de fatores intervenientes no padrão de consumo dos serviços públicos de saúde, imbricados entre si, que resultam em um quadro de "desigualdades cumulativas", geográficas e sociais. Os autores ressaltam a distância ainda existente entre o texto legal, de universalidade e equidade, da realidade encontrada.

Os resultados encontrados trazem alguns dados muito positivos como o aumento da assistência de alta complexidade; outros dados trazem consigo uma verdadeira tragédia assistencial como é o caso da redução dos leitos e internações na clínica médica com estabilização 

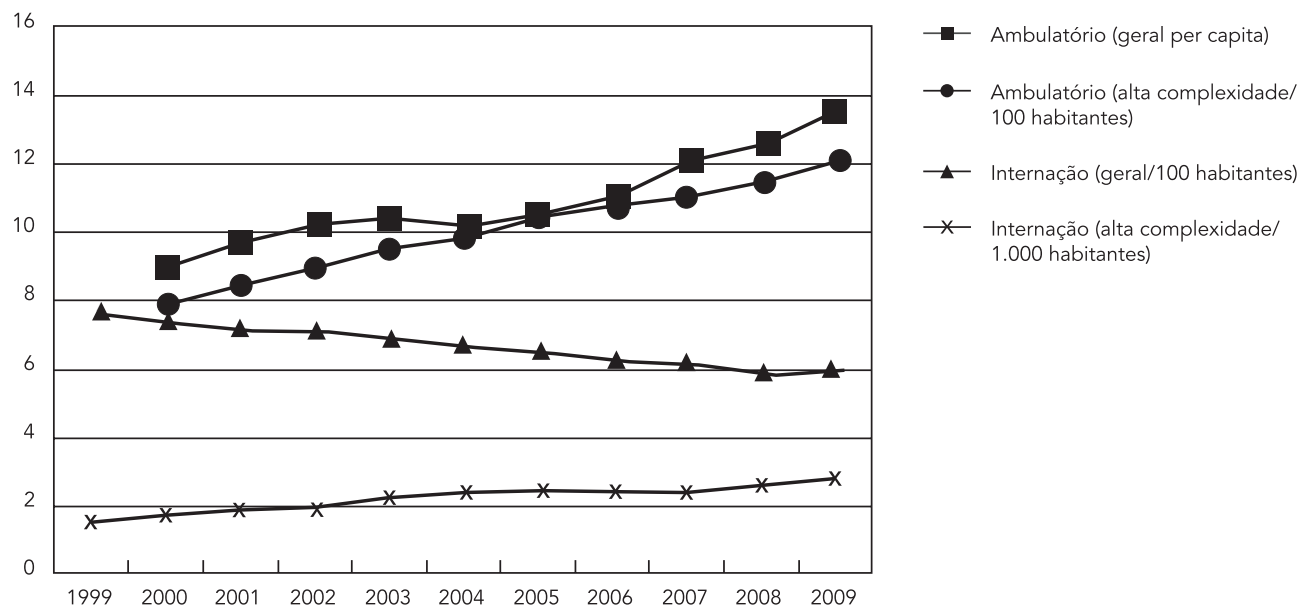

Fonte: Sistema de Informações Ambulatoriais/Sistema de Informações Hospitalares, Ministério da Saúde.

no número de idosos internados num momento de crescimento da população adulta e idosa. Segundo Wong \& Carvalho ${ }^{23}$, é nas idades mais avançadas que os cuidados com a saúde tornamse mais necessários e onerosos.

O perfil demográfico caracterizado por uma grande população em idade ativa, mas com parte dela sem a adequada inserção econômica, contribuindo para o aumento da violência; e o aumento da expectativa de vida ao nascer; que amplia o número de idosos, traz como consequência maior demanda por atendimentos, sobretudo de urgências e emergências por traumatismos, acidentes vasculares cerebrais e emergências cardiológicas. Responsáveis pela superlotação das emergências de todo país em busca de solução para seu problema de saúde que, aliada à baixa oferta de leitos de retaguarda, particularmente de clínica médica e especialidades, leva a situações desumanas - cidadãos que passam dias e até semanas em macas nos corredores das emergências do país 24 .

A assistência pediátrica é muito sensível às mudanças sociais e epidemiológicas. A redução da mortalidade infantil, a queda da natalidade e fecundidade e a diminuição da população menor de um ano determinam um maior cuidado das famílias com suas crianças, assim como a melhor assistência à saúde pelo Estado. A redução das internações pediátricas, como vimos, vem corroborar com o sucesso da priorização na as- sistência à saúde desse grupo etário, refletida não só na redução da mortalidade como também na morbidade entre as crianças.

A redução dos nascimentos também reflete diretamente na assistência obstétrica, diminuindo o número de partos realizados. Contudo, essa grande redução vem ocorrendo na rede pública com aumento do número de nascimentos na rede hospitalar privada (SINASC; http://www.ta bnet.datasus.gov.br/cgi/tabcgi.exe?sinasc/cnv/ nv, acessado em 11/Ago/2010), diminuindo a participação do SUS na assistência ao parto. Semelhante comportamento ocorre, provavelmente, em decorrência da melhoria das condições econômicas da população, possibilitando a contratação de planos de saúde, mas também deve ser considerada a má qualidade da assistência da rede pública, forçando a população a buscar alternativas assistenciais.

Os resultados contraditórios, discorridos anteriormente por este estudo, demonstram que, além da transição demográfica e epidemiológica, o Brasil também passa por um momento de transição do modelo assistencial. A transição epidemiológica é caracterizada pela diminuição da mortalidade pelas doenças infecciosas, o crescimento das doenças crônicas degenerativas e dos distúrbios mentais 10, além do crescimento das causas externas, com os homicídios substituindo as doenças infecciosas dos adultos nas áreas mais pobres das regiões metropolita- 
nas 25. A transição assistencial é caracterizada pelo aumento das ações e serviços ambulatoriais e pela redução das necessidades de internações pediátricas e obstétricas e pelo aumento das necessidades de clínica médica, clínica cirúrgica e reabilitação, em decorrência do crescimento da população adulta e idosa, que dependendo das condições sociais, pode ter menores exigências assistenciais. Ainda mais por apresentar, no Brasil, um "crescimento demográfico da terceira idade”, caracterizado por uma não predominância dos "idosos muito velhos" 10 .

\section{Resumo}

Este estudo avalia a assistência hospitalar e ambulatorial e sua capacidade de responder às novas exigências neste contexto da transição demográfica brasilei$\mathrm{ra}$. As informações foram obtidas dos estudos do IBGE, e sistemas de informações assistenciais do SUS (CNES, SIH e SIA). A redução das taxas de natalidade, fecundidade, mortalidade infantil e aumento da expectativa de vida ao nascer determinam ainda um crescimento demográfico, com redução da taxa de dependência, o que permite uma oportunidade para promover ajustes necessários. Entre 1999 e 2009, a população cresceu em mais 27,5 milhões de habitantes com redução de 26,7\% dos leitos e 947 mil internações com distorções na distribuição por clínicas, mas com aumento da assistência de alta complexidade ambulatorial e hospitalar. Os resultados demonstram que vivemos um momento de transição do modelo assistencial que exige maior capacidade de planejamento do futuro da assistência à saúde, tornando mais complexa a rede assistencial e repensando o modelo de atenção à saúde, preparandose para o grande crescimento da população idosa nas próximas décadas.

Assistência à Saúde; Transição Demográfica; Necessidades e Demandas de Serviços de Saúde
Portanto, vale ressaltar que se junto a essas características epidemiológicas e demográficas combinar-se um maior crescimento econômico, com distribuição de renda e uma ascensão social da população no país, poder-se-á ter aliado ao "bônus demográfico" um "bônus assistencial". Essas melhores condições sociais e um crescimento não tão grande das necessidades assistenciais permitiriam uma maior capacidade de planejamento do futuro da assistência à saúde, tornando mais complexa a rede assistencial e preparando-a para o grande crescimento da população idosa com predominância dos "idosos muito velhos" nas próximas décadas.

\section{Colaboradores}

A. C. G. Mendes, D. A. Sá, G. M. D. Miranda, T. M. Lyra e R. A. W. Tavares realizaram contribuições em todas as etapas do artigo, desde a sua concepção, análise e interpretação dos dados e redação. 


\section{Referências}

1. Yunes J. A dinâmica populacional dos países desenvolvidos e subdesenvolvidos. Rev Saúde Pública $1971 ; 5: 129-50$.

2. Ministério da Saúde. Estimativas de mortalidade infantil por microrregiões e municípios. Resumo da pesquisa. http://tabnet.datasus.gov.br/cgi/ mortinf/midescr.htm (acessado em 15/Set/2010).

3. Paiva PTA, Wajnman S. Das causas às consequências econômicas da transição demográfica. Rev Bras Estud Popul 2005; 22:303-22.

4. Instituto Brasileiro de Geografia e Estatística. Projeção da população do Brasil por sexo e idade. 1980-2050. Rio de Janeiro: Instituto Brasileiro de Geografia e Estatística; 2008.

5. Carvalho DM. Grandes sistemas nacionais de informações em saúde: revisão e discussão da situação atual. Inf Epidemiol SUS 1997; 6:7-46.

6. Mendes ACG, Silva Júnior JB, Medeiros KR, Lyra TM, Melo Filho DA, Sá DA. Avaliação do Sistema de Informações Hospitalares como fonte complementar na vigilância e monitoramento de doenças de notificação compulsória. Inf Epidemiol SUS 2000; 10:67-86

7. Scatena JHG, Tanaka OY. Utilização do Sistema de Informações Hospitalares (SIH-SUS) e do Sistema de Informações Ambulatoriais (SIA-SUS) na análise de descentralização da saúde em Mato Grosso. Inf Epidemiol SUS 2001; 10:19-30.

8. Veras RP. Considerações acerca de um jovem país que envelhece. Cad Saúde Pública 1988; 4:382-97.

9. Kalache A, Veras RP, Ramos LR. O envelhecimento da população mundial. Um desafio novo. Rev Saúde Pública 1987; 21:200-10.

10. Chaimowicz FA. Saúde dos idosos brasileiros às vésperas do século XXI: problemas, projeções e alternativas. Rev Saúde Pública 1997; 31:184-200.

11. Siqueira AB, Cordeiro RC, Perracini MR, Ramos LR. Impacto funcional da internação hospitalar de pacientes idosos. Rev Saúde Pública 2004; 38:687-94.

12. Instituto Brasileiro de Geografia e Estatística. Síntese de indicadores sociais. Uma análise das condições de vida da população brasileira. Rio de Janeiro: Instituto Brasileiro de Geografia e Estatística; 2008.

13. Brito F. Transição demográfica e desigualdades sociais no Brasil. Rev Bras Estud Popul 2008; 25:5-26.

14. Carvalho JAM, Rodríguez-Wong LL. A transição da estrutura etária da população brasileira na primeira metade do século XXI. Cad Saúde Pública 2008; 24:597-605.

15. Instituto Brasileiro de Geografia e Estatística. Pesquisa Nacional por Amostra de Domicílios. Um panorama da saúde no Brasil. Acesso e utilização dos serviços, condições de saúde e fatores de risco e proteção à saúde. Rio de Janeiro: Instituto Brasileiro de Geografia e Estatística; 2010.
16. Ministério da Saúde. Portaria no. 1101/GM. Estabelece os parâmetros de cobertura assistencial no âmbito do Sistema Único de Saúde - SUS. http:// www.dtr2001.saude.gov.br/sas / PORTARIAS / Port2002/Gm/GM-1101.htm (acessado em 01/ Ago/2010).

17. Conselho Nacional de Secretários de Saúde. Nota técnica nº. 26. Unificação das Tabelas do SIA e do SIH/SUS. Brasília: Conselho Nacional de Secretários de Saúde; 2006.

18. Castro MSM, Travassos C, Carvalho MS. Efeito da oferta de serviços de saúde no uso de internações hospitalares no Brasil. Rev Saúde Pública 2005; 39:277-84.

19. Wennberg JE. Time to tackle unwarranted variations in practice. BMJ 2011; 342:d1513.

20. Mendes ACG, Sá DA, Lyra TM, Lessa FJD. As doenças crônicas não transmissíveis e o acesso à assistência de alta complexidade: uma análise através dos sistemas de informações assistenciais. In: Freese E, organizador. Epidemiologia, políticas e determinantes das doenças crônicas não transmissíveis no Brasil. Recife: Editora Universitária UFPE; 2006. p. 105-19.

21. Berenstein C. O perfil etário dos custos de internação na saúde pública no Brasil: uma análise para as capitais das regiões metropolitanas do Brasil em 2000 [Dissertação de Mestrado]. Belo Horizonte: Centro de Desenvolvimento e Planejamento Regional de Minas Gerais, Universidade Federal de Minas Gerais; 2005.

22. Travassos C, Viacava F, Almeida MC. Desigualdade geográfica e sociais na utilização de serviços de saúde no Brasil. Ciênc Saúde Coletiva 2000; 23:133-49.

23. Wong LLR, Carvalho JA. O rápido processo de envelhecimento populacional do Brasil: sérios desafios para as políticas públicas. Rev Bras Estud Popul 2006; 23:5-26.

24. Mendes ACG. A qualidade da assistência de urgência e emergência: triangulando métodos e resultados. In: Mendes ACG, organizador. Delicadeza esquecida - avaliação da qualidade das emergências. Recife: Editora Universitária UFPE; 2010. p. 105-19.

25. Paes-Souza R. Diferenciais intraurbanos de mortalidade em Belo Horizonte, Minas Gerais, Brasil, 1994: revisitando o debate sobre transições demográfica e epidemiológica. Cad Saúde Pública 2002; 18:1411-21.

Recebido em 06/Out/2010

Versão final reapresentada em 22/Nov/2011

Aprovado em 31/Jan/2012 